\title{
Indoor Radio Propagation Model Based on Dominant Path
}

\author{
Yongxiang Zhao ${ }^{1}$, Meifang $\mathrm{Li}^{2}$, Feng $\mathrm{Shi}^{3}$ \\ ${ }^{1}$ Wuhan University, State Key Laboratory of Software Engineering, Wuhan, China \\ ${ }^{2}$ Wuhan University of Technology, School of Management, Wuhan, China \\ ${ }^{3}$ Wuhan Academy of Social Sciences, Wuhan, China \\ Email: zhaosanhe@263.net,poplimeif@126.com, sf196293@163.com \\ Received November 18, 2009; revised December 22, 2009; accepted January 12, 2010
}

\begin{abstract}
When there are bigger obstacles in the indoor environment such as elevator, the radio waves basically can not penetrate it. The contribution of received signal strength by transmission and reflection will be greatly reduced, and most of the time, the radio waves will reach the user by bypass diffraction. Therefore, the traditional path loss model is no longer applicable, and the improved model should be proposed. In this paper, we firstly proposed an indoor radio propagation model based on dominant path in which the received signal strength has nothing to do with the direct distance between user and access point, but is related to the length of dominant path. Then on the basis of dominant path model, the NLOS influence is considered in order to further improve the accuracy of dominant path model. Experimental results demonstrated that the proposed dominant path model can improve the accuracy of traditional path loss model remarkably.
\end{abstract}

Keywords: Wireless LANs, Indoor Positioning, Radio Waves, Received Signal Strength, Path Loss Model, Dominant Path

\section{Introduction}

Indoor positioning systems have become very popular in recent years, both the research and commercial products in this area are new, and many people in academia and industry are currently involved in the development of these systems.

It is possible to obtain the position location of a mobile device in two ways: by using a special infrastructure or by improving the existing communications infrastructure. GPS is not suitable for indoor areas because of the lack of coverage. Therefore, it is preferable to employ the existing wireless communications infrastructure to determine the location of users. The wireless communications infrastructure is primarily based on wireless local area networks (WLANs) [1] in indoor areas. Thus, these indoor positioning systems mainly lie on an IEEE $802.11 \mathrm{~b}$ or $802.11 \mathrm{~g}$ WLAN.

Owing to the harsh multi-path environment in indoor areas, techniques that use triangulation or direction are not very attractive and often can yield highly erroneous results [2]. Location fingerprinting refers to techniques that match the fingerprint of some characteristic of the signal that is location dependent. In WLANs, an easily available signal characteristic is the received signal strength (RSS) and this has been used in [3] for fingerprinting.

Due to severe multi-path fading and shadowing present in the indoor environment, there is no accurate indoor radio propagation model. A traditional radio propagation model, named path loss model, is often used to describe the characteristic of indoor environment.

However, when there are bigger obstacles in the indoor environment such as elevator, the radio waves basically can not penetrate it. The contribution of received signal strength by transmission and reflection will be greatly reduced, and most of the time, the radio waves will reach the user by bypass diffraction. Therefore, the traditional path loss model is no longer applicable, and the improved model should be proposed.

This paper is organized as follows. In Section 2, we proposed three indoor radio propagation models, named Path Loss Model, Dominant Path Model 1 and Dominant Path Model 2. Section 3 described experimental testbed which is a typical office building environment. In Section 4, the experimental results of three models were discussed and analyzed. Section 5 depicted field strength simulation results of dominant path model 2 for the experimental access points. Finally, Section 6 summarized the paper and gave possible future research directions. 


\section{Three Indoor Radio Propagation Models}

\subsection{Path Loss Model}

In this paper, a path loss model [4] is introduced to describe the characteristic of indoor environment which is as follows:

$$
P(d)=P\left(d_{0}\right)-10 \times n \times \log \left(\frac{d}{d_{0}}\right)+\zeta
$$

In the Equation (1), the $P(d)$ is the received signal strength of users when the distance between users and access points is $d$. And $P\left(d_{0}\right)$ is the received signal strength of users when the distance between users and access points is $d_{0}$ ( $d_{0}$ is equal to 1 meter). The parameter $n$ is the path-loss index which depends upon the indoor propagation environment. $\zeta$ is the shadowing factor which is a random variable.

\subsection{Dominant Path Model 1}

When there are bigger obstacles in the indoor environment such as elevator, the radio waves basically can not penetrate it. The contribution of received signal strength by transmission and reflection will be greatly reduced, and most of the time, the radio waves will reach the user by bypass diffraction. Therefore, the traditional path loss model is no longer applicable because the received signal strength $P(d)$ has nothing to do with the direct distance $d$ between user and access point, but is related to the length $L$ of dominant path which is described in Figure 1.

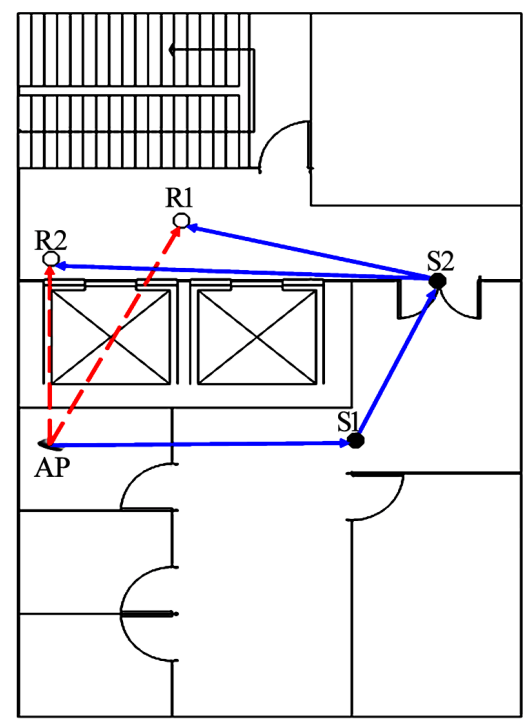

Figure 1. Dominant path model 1 in indoor scenarios.
In Figure 1, AP is placed in a small room, sampling points R1 and R2 are close to elevator. The traditional path loss model supposes that the radio waves from AP directly arrive at sampling points R1 and R2 by penetrating the elevator. However, the elevator is bigger obstacle which is made up of metal and concrete materials, and can not be penetrated by radio waves. In fact, the radio waves from AP reach the sampling points $\mathrm{R} 1$ and $\mathrm{R} 2$ by bypass diffraction, that is, the radio waves firstly reach the point $\mathrm{S} 1$, then reach the point $\mathrm{S} 2$, and finally arrive at points $\mathrm{R} 1$ and $\mathrm{R} 2$.

The proposed Dominant Path Model 1 was described as follows:

$$
P(L)=P\left(d_{0}\right)-10 n \log \left(\frac{L}{d_{0}}\right)
$$

In the Equation (2), the $P(L)$ is the received signal strength of users when the length of dominant path is $L$. And $P\left(d_{0}\right)$ is the received signal strength of users when the length of dominant path is $d_{0}$ ( $d_{0}$ is equal to 1 meter). The parameter $n$ is also the path-loss index which depends upon the indoor propagation environment.

\subsection{Dominant Path Model 2}

However, the received signal strength (RSS) in indoor environment is influenced by Non-line-of-sight (NLOS) propagation, and the attenuation level of signal strength is different when in Line-of-sight (LOS) environment and in Non-line-of-sight (NLOS) environment. Therefore, the LOS propagation and NLOS propagation should be discussed and analyzed separately in order to further improve the accuracy of dominant path model which is depicted in Figure 2.

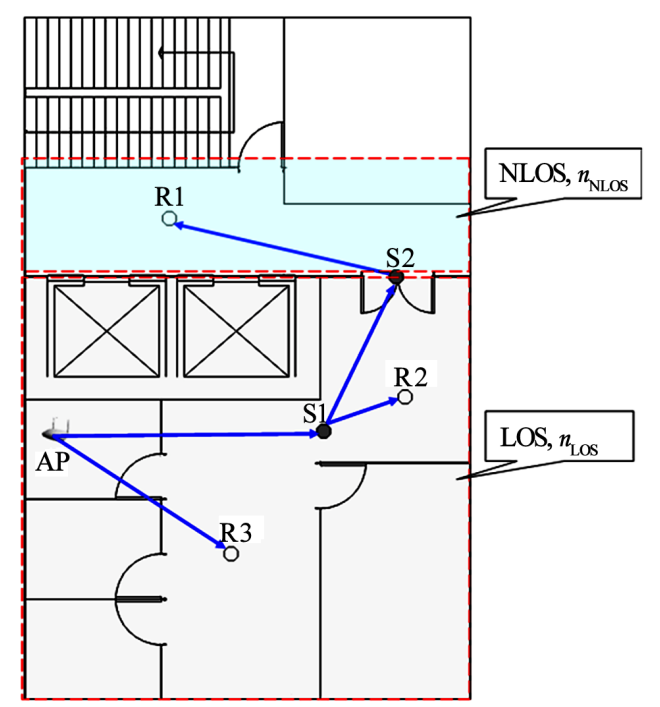

Figure 2. Dominant path model 2 in indoor scenarios. 
In Figure 2, the whole experimental environment is divided into two separate parts. 1) Room part. It includes three small rooms and three big rooms, so, the Room part is approximate LOS environment in which the path-loss index is $n_{L O S} .2$ ) Corridor part. All the APs are placed in the Room part, thus, the Corridor part is typical NLOS environment in which the path-loss index is $n_{N L O S}$ because the radio waves from APs can not penetrate thicker concrete wall.

The proposed Dominant Path Model 2 was described as follows:

$$
P(L)= \begin{cases}P\left(d_{0}\right)-10 n_{\mathrm{LOS}} \log \left(\frac{L}{d_{0}}\right), & \text { Line-of-sight(LOS) } \\ P\left(d_{0}\right)-10 n_{\mathrm{NLOS}} \log \left(\frac{L}{d_{0}}\right), & \text { Non-Line-of-sight(NLOS) }\end{cases}
$$

In the Equation (3), the $n_{\text {LOS }}$ is the path-loss index in LOS environment, and the $n_{N L O S}$ is the path-loss index in NLOS environment. Other parameters are the same as Dominant Path Model 1.

\section{Experimental Testbed}

The experimental testbed is located in the 11th floor of Cherry Blossom Building. The floor has dimensions of 15 meters by 10 meters and includes 6 rooms which is the typical indoor office environment. In this work, we choose the TP-LINK TL-WA501G as our experimental APs because of its low cost. The WLAN consists of four access points, and the MAC, SSID and operating channel of these access points are listed in Table $\mathbf{1}$.

The survey trail and AP placement of experimental testbed is shown in Figure 3. From Figure 3, we can see that our experimental testbed is the typical indoor office environment. The AP placement of experimental testbed refers to the conclusion of literature [5], that is, the access points should be scattered asymmetrically and should be placed around the site in a "zigzag" pattern rather than placing several APs close together or placing them on a straight line.

Table 1. MAC, SSID and channel of experimental access points.

\begin{tabular}{ccc}
\hline MAC & SSID & Channel \\
\hline 00:1D:0F:43:CA:7F & AP1 & 9 \\
00:1D:0F:43:CA:86 & AP2 & 13 \\
00:1D:0F:43:CB:A1 & AP3 & 3 \\
00:1D:0F:43:CB:A8 & AP4 & 1 \\
\hline
\end{tabular}

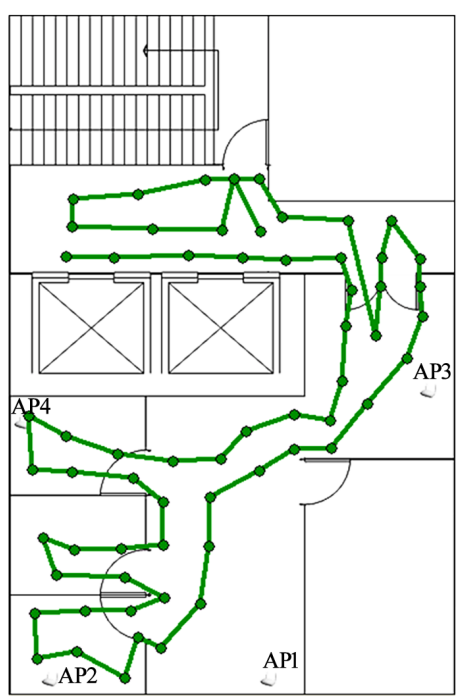

Figure 3. The survey trail and AP placement of experimental testbed.

The minimum distance between two locations or grid spacing was fixed at 1.5 meters. At each location, we calculated the average of 50 samples, and it would spend 25 seconds for each location when scanning frequency was set to two times per second.

\section{Experimental Results and Analysis}

In our experiments, $d_{0}$ is equal to 1 meter, and $P\left(d_{0}\right)$ is set to $-28.0 \mathrm{~dB}$. The RSS estimation results and RSS estimation error comparison for Path Loss Model, Dominant Path Model 1 and Dominant Path Model 2 are listed as follows.

\subsection{The RSS Estimation Results of Three Propagation Models}

1) The RSS Estimation Results of Path Loss Model

The RSS Estimation Results of Path Loss Model are shown in Figure 4, and the path-loss index of Path Loss Model for four APs is listed in Table 2.

From the experimental results of Figure 4 and Table 2,

Table 2. The path-loss index of path loss model.

\begin{tabular}{cc}
\hline AP Name & Path-loss Index $n$ \\
\hline AP1 & 3.45 \\
AP2 & 3.73 \\
AP3 & 3.20 \\
AP4 & 4.20 \\
\hline
\end{tabular}




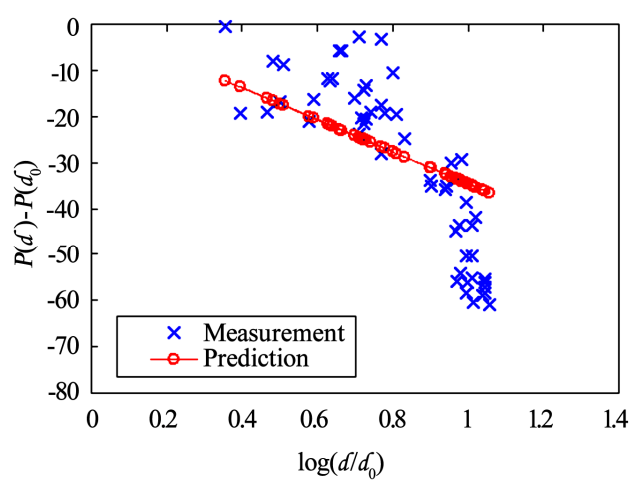

(a) The RSS estimation results of AP1

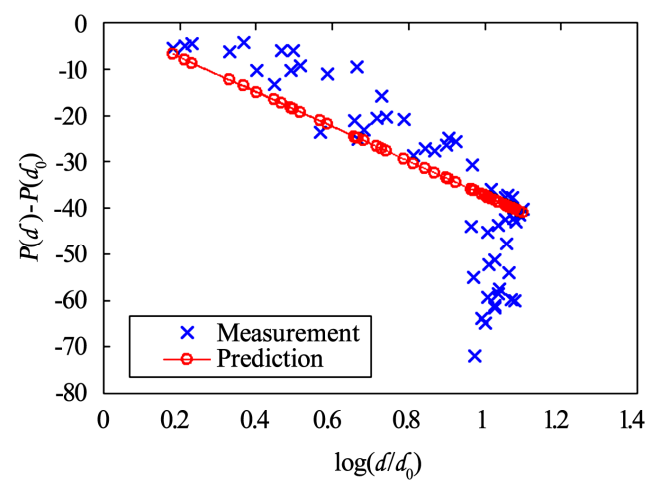

(b) The RSS estimation results of AP2

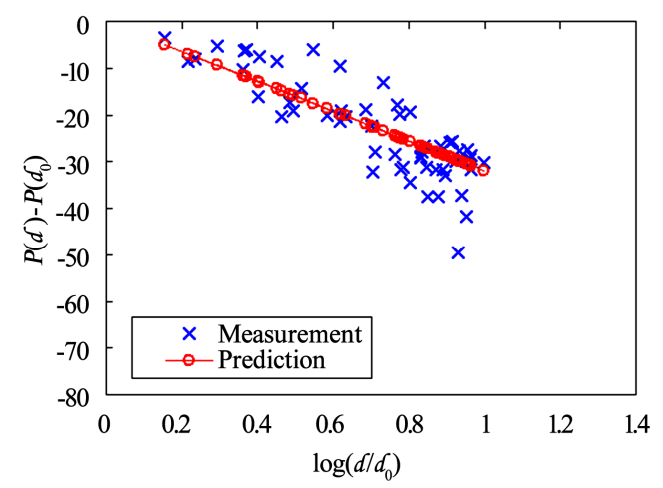

(c) The RSS estimation results of AP3

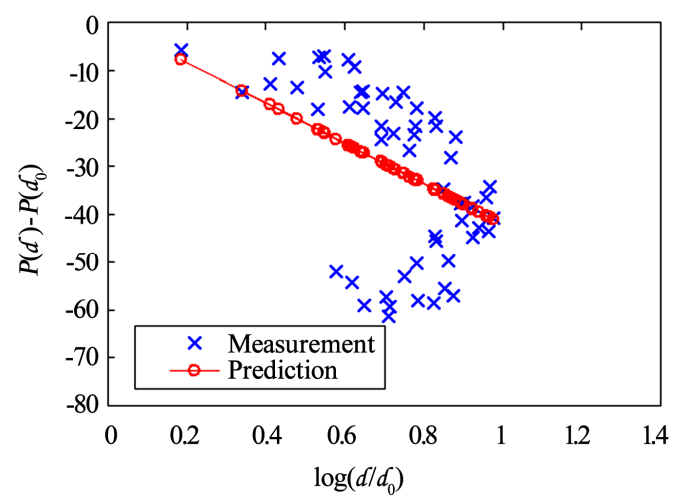

(d) The RSS estimation results of AP4

Figure 4. The RSS estimation results of path loss model. we can see that the RSS estimation result of AP3 is better, and its path-loss index is 3.20. However, the RSS estimation results of AP1, AP2 and AP4 are worse, and the path-loss index are 3.45, 3.73 and 4.20.

The reason is that, the AP3 is placed at the entrance of Room part, thus, the propagation environment of radio waves in Corridor part is close to the propagation environment in Room part which is approximate LOS environment. But the AP1, AP2 and AP4 are placed in the inside room which are farther away from the corridor and elevator, thus, the attenuation level of signal strength in Corridor part is more serious than in Room part.

The most serious situation is that, from Figure 4(b) and Figure 4(d), we can see that the radio waves propagation does not match with the Path Loss Model at all in Corridor part because the received signal strength is weaker and weaker when the direct distance between user and AP is closer and closer. Therefore, the traditional path loss model is no longer applicable because the received signal strength has nothing to do with the direct distance between user and access point, but is related to the length of dominant path.

2) The RSS Estimation Results of Dominant Path Model 1

The RSS Estimation Results of Dominant Path Model 1 are shown in Figure 5, and the path-loss index of Dominant Path Model 1 for four APs is listed in Table 3.

From the experimental results of Figure 5 and Table 3, we can see that the RSS estimation results of AP1, AP2, AP3 and AP4 are all better, and the path-loss index are 3.26, 3.48, 3.10 and 3.43.

Compared with the result of Path Loss Model, the Dominant Path Model 1 achieves remarkable improvement because the path-loss index of four APs are all decreased, for example, the path-loss index of AP1 decreases to 3.26 from 3.45, the path-loss index of AP2 decreases to 3.48 from 3.73, the path-loss index of AP3 decreases to 3.10 from 3.20, and the path-loss index of AP4 decreases to 3.43 from 4.20. Therefore, the Dominant Path Model 1 is more rational and accurate than the traditional Path Loss Model.

3) The RSS Estimation Results of Dominant Path Model 2

The RSS Estimation Results of Dominant Path Model 2 are shown in Figure 6, and the path-loss index of Dominant Path Model 2 for four APs is listed in Table 4.

Table 3. The path-loss index of dominant path model 1.

\begin{tabular}{cc}
\hline AP Name & Path-loss Index $n$ \\
\hline AP1 & 3.26 \\
AP2 & 3.48 \\
AP3 & 3.10 \\
AP4 & 3.43 \\
\hline
\end{tabular}




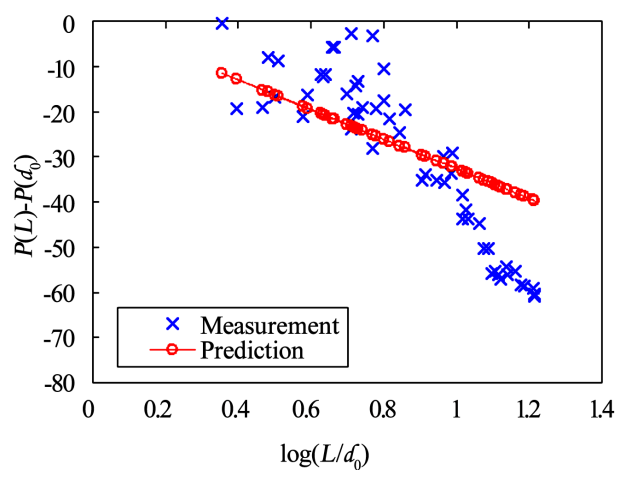

(a) The RSS estimation results of AP1

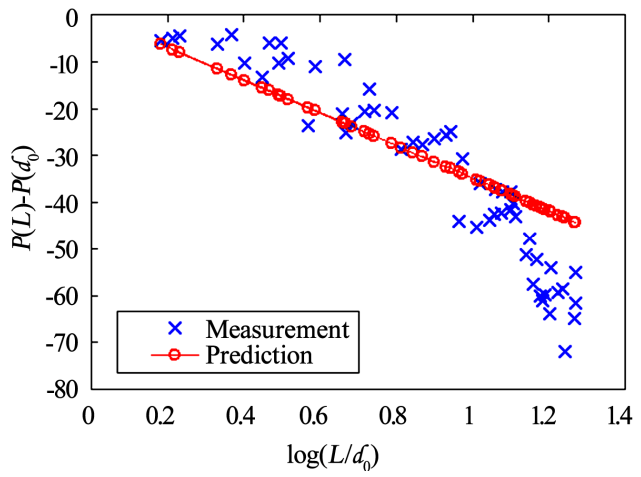

(b) The RSS estimation results of AP2

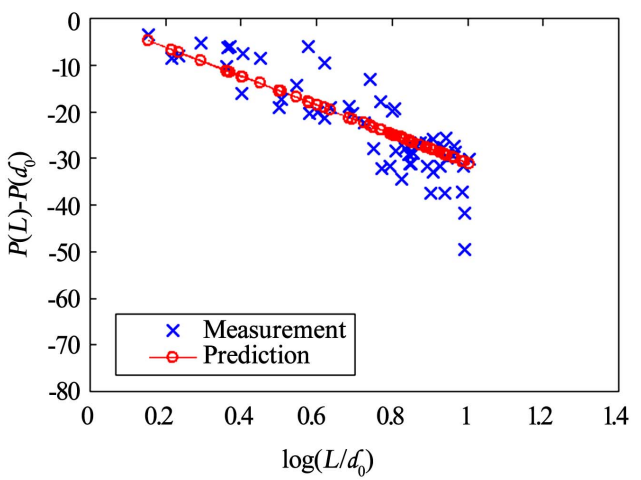

(c) The RSS estimation results of AP3

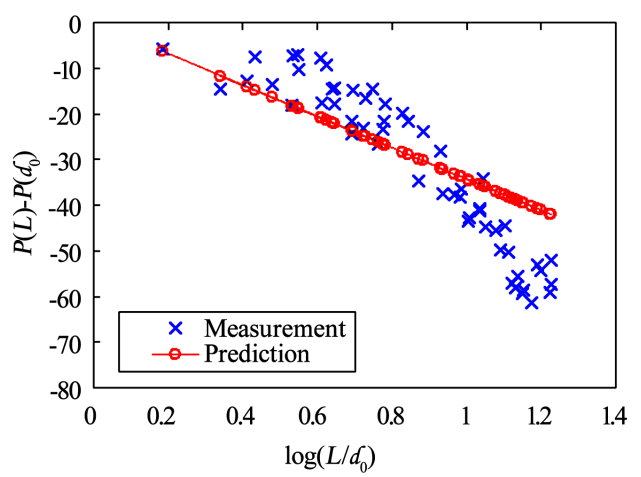

(d) The RSS estimation results of AP4

Figure 5. The RSS estimation results of dominant path model 1.

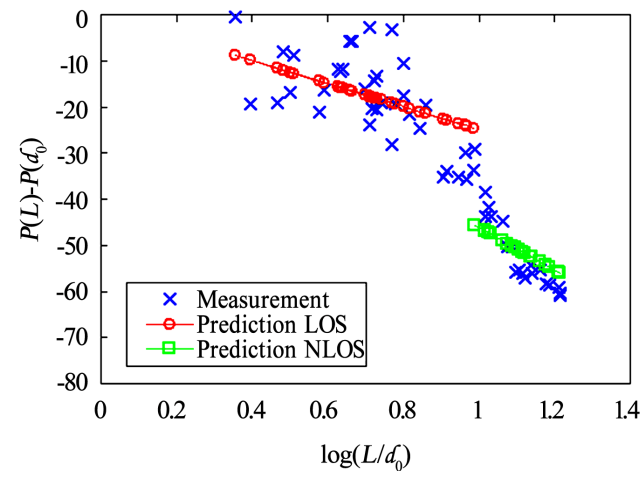

(a) The RSS estimation results of AP1

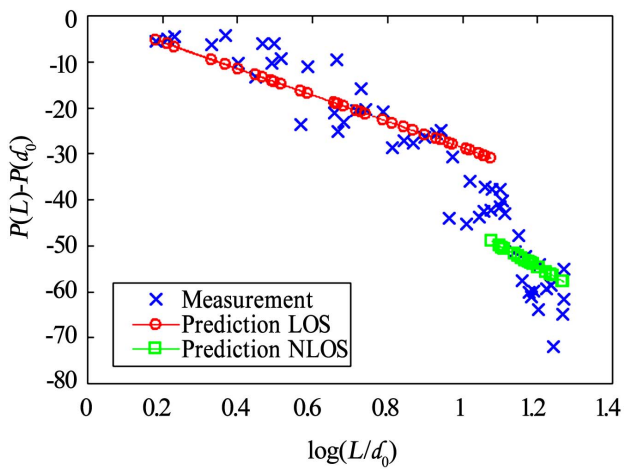

(b) The RSS estimation results of AP2

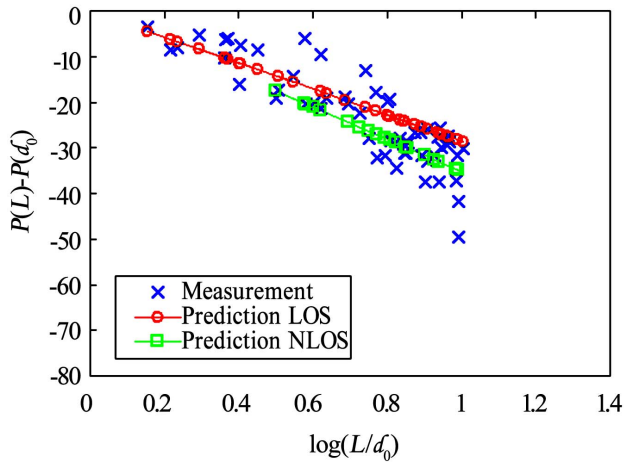

(c) The RSS estimation results of AP3

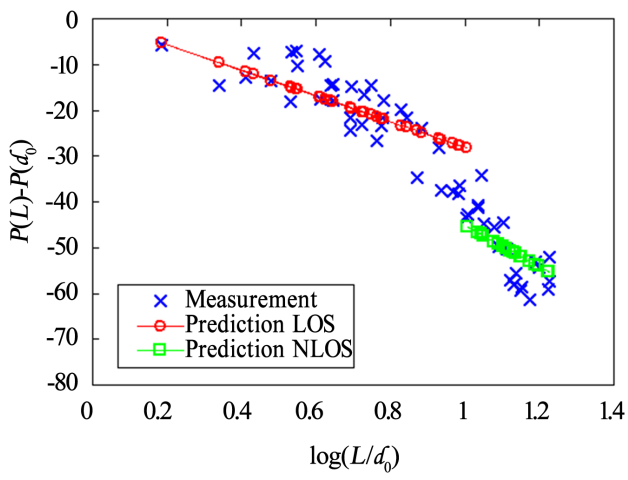

(d) The RSS estimation results of AP4

Figure 6. The RSS estimation results of dominant path model 2. 
Table 4. The path-loss index of dominant path model 2.

\begin{tabular}{ccc}
\hline AP Name & Path-loss Index $n_{\text {LOS }}$ & Path-loss Index $n_{\text {NLOS }}$ \\
\hline AP1 & 2.49 & 4.62 \\
AP2 & 2.87 & 4.56 \\
AP3 & 2.86 & 3.52 \\
AP4 & 2.81 & 4.52 \\
\hline
\end{tabular}

From the experimental results of Figure 6 and Table 4, we can see that the RSS estimation results of AP1, AP2, AP3 and AP4 achieve further improvement after the LOS propagation and NLOS propagation are analyzed separately.

The path-loss index $n_{\mathrm{LOS}}$ of four APs are 2.49, 2.87, 2.86 and 2.81 which are all less than 3 , and the path-loss index $n_{\mathrm{NLOS}}$ of four APs are 4.62, 4.56, 3.52 and 4.52. Therefore, the Dominant Path Model 2 is more rational and accurate than the Dominant Path Model 1.

\subsection{The RSS Estimation Error Comparison of Three Propagation Model}

The RSS estimation error comparison of three propagation model, named Path Loss Model, Dominant Path Model 1 and Dominant Path Model 2 , are shown in Figure 7 , and the RSS estimation error values of three propagation model are listed in Table 5.

Experimental results of Figure 7 and Table 5 demonstrated that the accuracy of Path Loss Mode is worst, and its average mean value for four APs is 9.32, its average

Table 5. The RSS estimation error values of three propagation model.

\begin{tabular}{ccccccc}
\hline & \multicolumn{2}{c}{$\begin{array}{c}\text { Path Loss } \\
\text { AP }\end{array}$} & \multicolumn{2}{c}{ Model (dB) } & \multicolumn{2}{c}{$\begin{array}{c}\text { Dominant Path } \\
\text { Name }\end{array}$} \\
\cline { 2 - 7 } & $\begin{array}{c}\text { Mean } \\
\text { Value }\end{array}$ & Std. Dev. & $\begin{array}{c}\text { Mominant Path } \\
\text { Malue } \\
\text { vodel 2 (dB) }\end{array}$ & Std. Dev. & $\begin{array}{c}\text { Mean } \\
\text { value }\end{array}$ & Std. Dev. \\
\hline AP1 & 11.06 & 7.78 & 10.25 & 6.76 & 5.64 & 4.03 \\
AP2 & 9.57 & 8.15 & 8.12 & 6.68 & 5.61 & 4.33 \\
AP3 & 4.46 & 3.79 & 4.19 & 3.62 & 3.51 & 3.22 \\
AP4 & 12.20 & 8.77 & 8.25 & 5.55 & 4.76 & 3.58 \\
Average & 9.32 & 7.12 & 7.70 & 5.65 & 4.88 & 3.79 \\
\hline
\end{tabular}

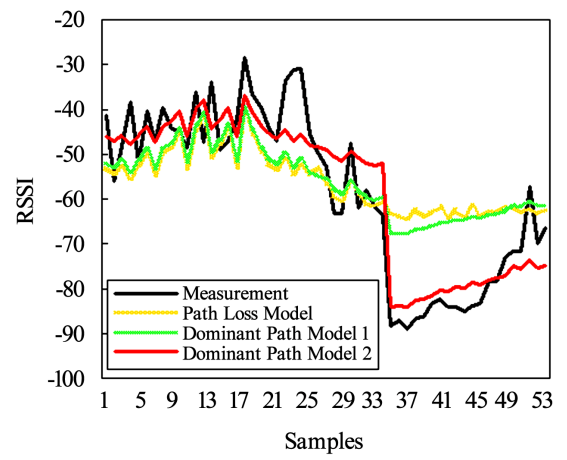

(a) The RSS error comparison of AP1

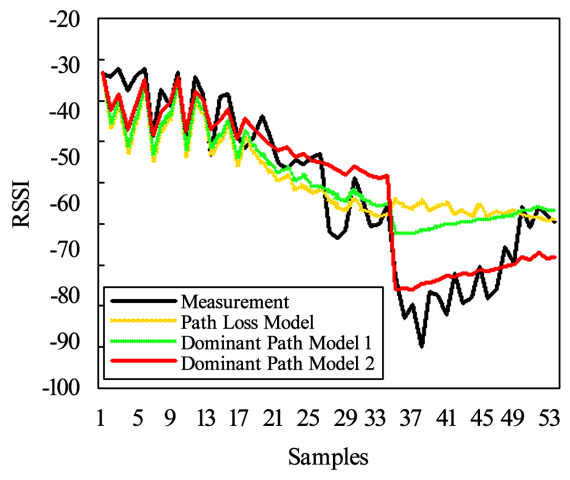

(b) The RSS error comparison of AP2

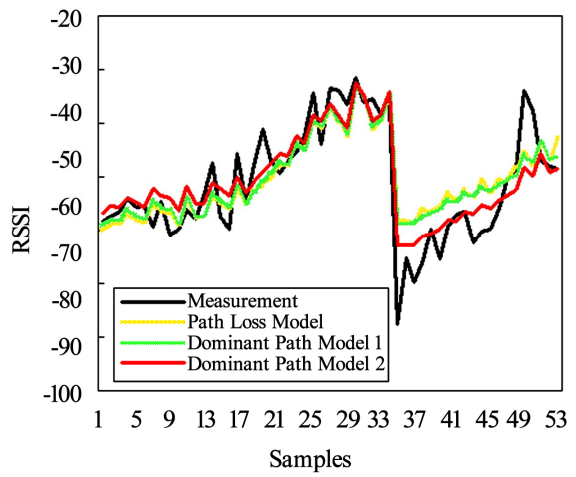

(c) The RSS error comparison of AP3

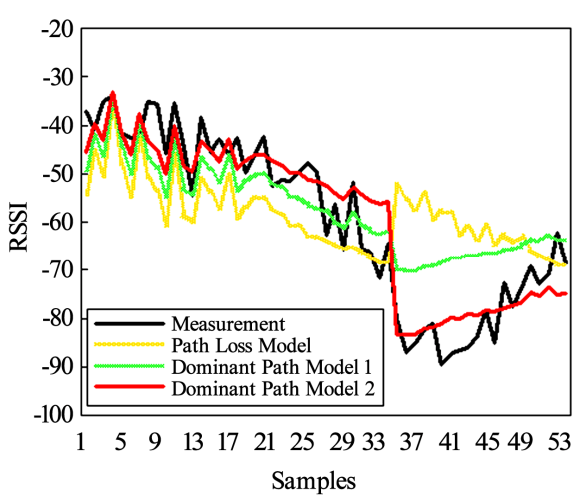

(d) The RSS error comparison of AP4

Figure 7. The RSS estimation error comparison of three propagation model. 
standard deviation is 7.12. The accuracy of Dominant Path Model 1 is better, and its average mean value for four APs is 7.70, its average standard deviation is 5.65. Finally, the accuracy of Dominant Path Model 2 is best, and its average mean value for four APs is only 4.88, its average standard deviation is only 3.79 .

Therefore, the proposed dominant path model in this paper can improve the accuracy of traditional path loss model remarkably. When the NLOS influence is further considered, the dominant path model is more rational and accurate, and its average estimation error of received signal strength is only $4.88 \mathrm{~dB}$ in the typical indoor office environment.

\section{The Field Strength Simulation of Dominant Path Model}

Finally, we depicted the whole field strength simulation

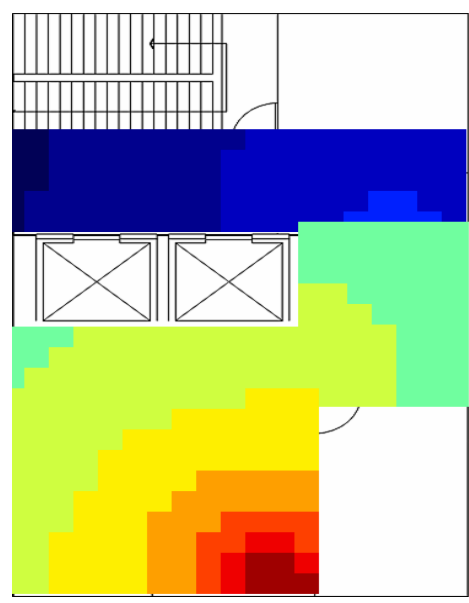

(a) Field strength simulation of AP1

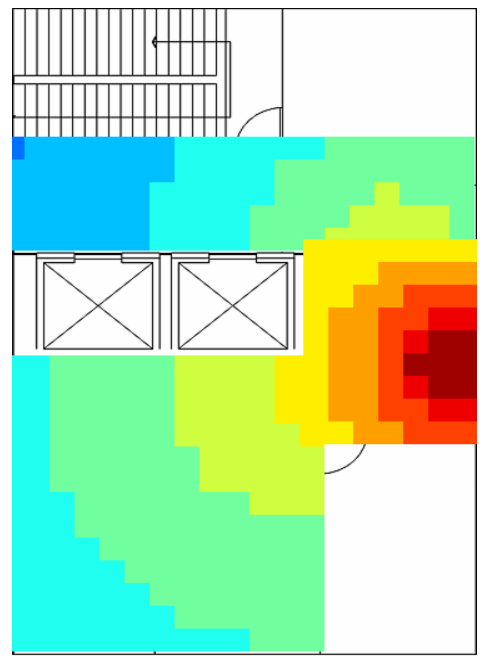

(c) Field strength simulation of AP3 results of dominant path model 2 for the experimental access points which is shown in Figure 8. In this paper, the color indication of received signal strength is as follows:

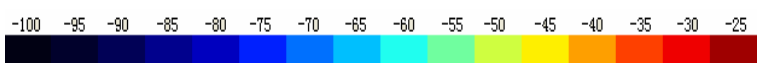

The field strength simulation of Figure 8 demonstrated that when there are bigger obstacles in the indoor environment such as elevator, the radio waves basically can not penetrate it. The contribution of received signal strength by transmission and reflection will be greatly reduced, and most of the time, the radio waves will reach the user by bypass diffraction.

In dominant path model 2, the received signal strength has nothing to do with the direct distance between user and access point, but is related to the length of dominant path. Therefore, the proposed dominant path model in

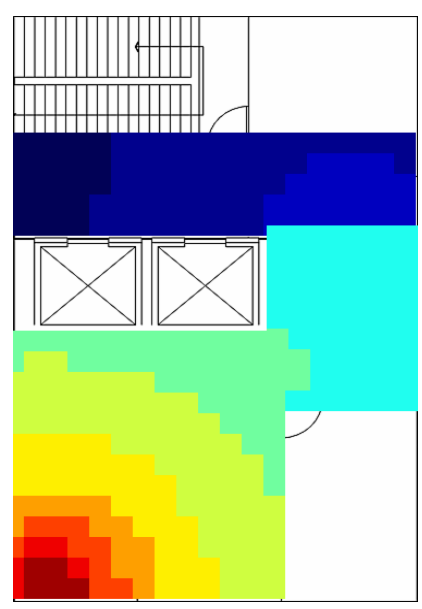

(b) Field strength simulation of AP2

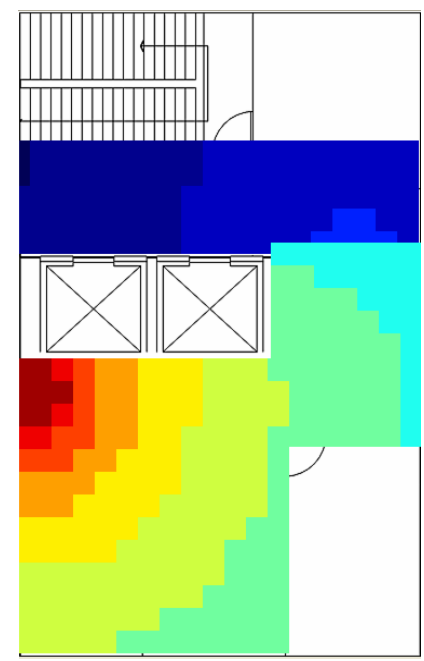

(d) Field strength simulation of AP4

Figure 8. The field strength simulation of dominant path model 2. 
this paper can improve the accuracy of traditional path loss model remarkably.

\section{Conclusions and Future Work}

In this paper, we firstly proposed an indoor radio propagation model based on dominant path in which the received signal strength has nothing to do with the direct distance between user and access point, but is related to the length of dominant path. Then on the basis of dominant path model, the NLOS influence is considered in order to further improve the accuracy of dominant path model. Experimental results demonstrated that the proposed dominant path model can improve the accuracy of traditional path loss model remarkably.

Future research directions are as follows:

1) Further improve the proposed dominant path model in this paper, for example, the influence factor of wall should be further considered.

2) Study and design intelligent algorithm to automatically search and determine the dominant path of radio propagation in bigger office building environment in order to further improve the applicability and scalability of the proposed dominant path model in this paper.

\section{Acknowledgment}

This work was supported by National 863 project (Grant Nos. 2007AA12Z324, 2009AA12Z324).

\section{References}

[1] IEEE P802.11 Working Group, "Changes and additions to IEEE 802.11,” 1999 Edition, January 2000.

[2] J. Caffery Jr. and G. Stuber, “Overview of radiolocation in CDMA cellular systems," IEEE Communications Magazine, April 1998.

[3] M. A. Youssef, A. Agrawala, A. U. Shankar, and S. H. Noh, "A probabilistic clustering-based indoor location determination system,” Technical Report CS-TR-4350 and UMIACS-TR-20002-30, University of Maryland, 2002.

[4] T. S Rappaport, "Wireless communications principles and practices $[\mathrm{M}]$," Second Edition, Publishing House of Electronics Industry, 2004.

[5] Y. X. Zhao, H. B. Zhou, M. F. Li, "Indoor access points location optimization using differential evolution,” In International Conference on Computer Science and Software Engineering, CSSE '08, IEEE Computer Society, pp. 382-385, 2008. 